\title{
A Sustainable Model of Function Decomposition Based on Effect
}

\author{
Hongxun Liu ${ }^{1, *}$, Zhu $\mathrm{Huo}^{2}$, Guozhong Cao ${ }^{1}$, Chengye Zhang ${ }^{1}$, and Shumin Zhang $^{1}$ \\ ${ }^{1}$ School of Mechanical Engineering, Hebei University of Technology \\ 300130, Tianjin, P.R. China \\ ${ }^{2}$ Clean Technology Development Office, Administrative Center for China's Agenda 21 \\ 100038, Beijing, P. R. China \\ jingshiji86@126.com
}

\begin{abstract}
Function decomposition is an important step in the process of product design, in both original design and redesign. Following "Three-Flows Principle" complies with objective rules, and the result of product design proves its importance. For the many-to-many relationship between function and effect, when different effect is selected for a function, flows will be very different. The sustainability of product has close relation with the functions, principles, structure and material of product. In the process of function decomposition, designer should follow the sustainable design criteria for effects. Besides, it is significant to unearth available resources in function decomposition. A model of functional decomposition based on effect followed the sustainable design criteria and "Three-Flows Principle" is established in this article. Last, functional structure of heating equipment is established following both the new model and existing model. The results prove the superiority of the new model.
\end{abstract}

Keywords: function decomposition, effect, sustainability, available resources.

\section{Introduction}

Currently, the descriptions of functional structure are function tree and functional chain. There are many methods to establish function tree and two have good effect among them. One is a top-down approach, Function Analysis System Technique method [1]. Following this method, the function tree is depended on the experience of the designer. Another is a bottom-up approach, the Subtract and Operate Procedure [2]. The underlying assumption to use this method is that either a form concept or actual product exists. So it limits the chance to use it. The description of functional structure with the integration of function tree and functional chain is based on the operation analysis of flows. Flows are physical phenomena, which are, material, energy, and signal, intrinsic to the product function. So following "Three-Flows Principle" complies with objective rules, and the result of product design proves its importance. But, the results of patent analysis in TRIZ prove the many-to-many relationship between function and effect [3]. When different effect is selected for a

* 1982-, master degree candidate. His research interests: innovative design.

D. Cavallucci, R. De Guio, and G. Cascini (Eds.): CAI 2011, IFIP AICT 355, pp. 1-6, 2011.

(C) IFIP International Federation for Information Processing 2011 
function, the flows will be very different. So the functional structure will be very experiential, without selecting the effect in the process based on "Three-Flows Principle". Secondly, the sustainability of product has close relation with the functions, principles, structure and material of product [4]. Moreover, it is difficult to improve the efficiency of the selected principle through subsequent selection of functions, structure and material.

The overall function is realized by a group of sub-functions organized with some relations. Sub-functions and relations constitute the functional structure, which not only describes the movement of flows, but also shows the relations between subfunctions. For convenient exchange, drawing functional structure should follow standard signs. The standard signs see Fig.1.

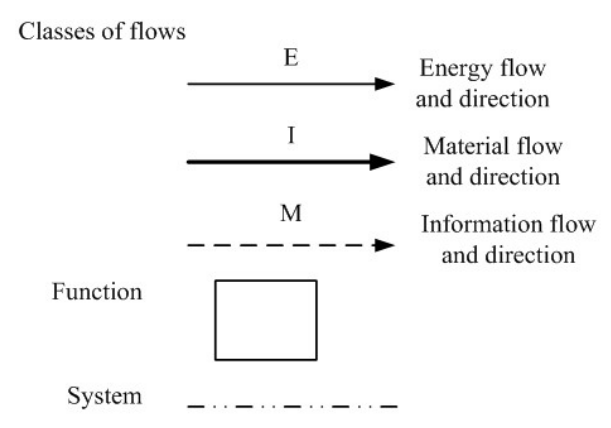

Fig. 1. Signs for functional structure

\section{Function Decomposition}

The purpose of function decomposition is to describe product on functional level. It will be significant to build a library of available resources [5] before function decomposition. An important aspect is the compare between input flows and available resources to seek the transition route. Moreover, along with the process of functional decomposition the library increases, because more effects will be selected and more output flows are available resources or will be transformed into available resources. More adequately available resources are unearthed, that should follow first material object then virtual object, more innovativeness the product has.

There may be some restraint between sub-functions, which are not resolved in the analysis process based on flows. So it is necessary to optimize the functional structure, mainly following sustainable design criteria for effect and function, between which there may be contradictions which should be resolved under the help of the contradiction solving matrix or separation principles from TRIZ [6].

Designer can plan product's layout in physical structure, through the modularization of functional structure. The modularization should follow some principles:

1. Divide a group of sequential operating functions for a flow in a module as far as possible.

2. Divide the functions in a module which has high dependency.

3. Ensure the convenience of module in manufacture. 


\section{Sustainable Design Criteria}

The analyses of patents confirm the many-to-many relationship between function and effect.

In the one hand, different scientific principle has different flows, which's attribute determines the sustainability of effect. In the process of function decomposition, analyses of inputs and out puts are necessary, then add operation to ensure inputs can be got from available resources and outputs can be changed into available resources and with sustainability.

On the other hand, effects can be classified into produce energy, store energy, transform energy and utilize energy. In different effects the utilization ratio or transformation efficiency or production efficiency may be different. Furthermore, it is difficult to improve the efficiency of final products through subsequent selection of function, structure and material, if the energy efficiency of scientific principle selected is bad. So it is chiefly work to select sustainable effect for enhancing the sustainability of product.

In the process of function decomposition, selecting effects should follow the principles:

1. Try to choose principles with high energy efficiency.

2. Try to choose principles that need simple structure.

3. Avoid using the principle of electromagnetic radiation generated.

4. Avoid using the principle of environmental pollution produced.

5. Avoid using the principle of using or producing toxic substances.

6. Avoid using the principle of produces high risk of fire or explosion.

When combine functional structure, should follow some principles:

1. Functional structure should be as simple as possible. Less sub-functions as far as possible, in order to reduce the number of components.

2. Merge compatible functions.

3. Functional sharing principle, and avoid too complex components.

4. Multi-function principle, make the products and components with multiple functions.

5. Functional independent principle, allowing users to shutdown system partially or totally.

6. Avoid the functions that produce harmful material flows, add auxiliary functions to eliminate harmful material flows or change them into useful resources.

\section{Sustainable Model of Function Decomposition}

Fig. 2 is a process model of functional decomposition, following the "Three-Flows Principle" and sustainable design criteria. And in the Fig. 2, F (E) is the relationship between function and effect. The application process of this model is divided into nine steps: establish a library of available resources, decompose the overall function into primary functions, seek and select effects, analyze output flows, add operations, analyze input flows, describe functional structure, optimize functional structure and modularize functional structure. Some steps are repeated in the process. 


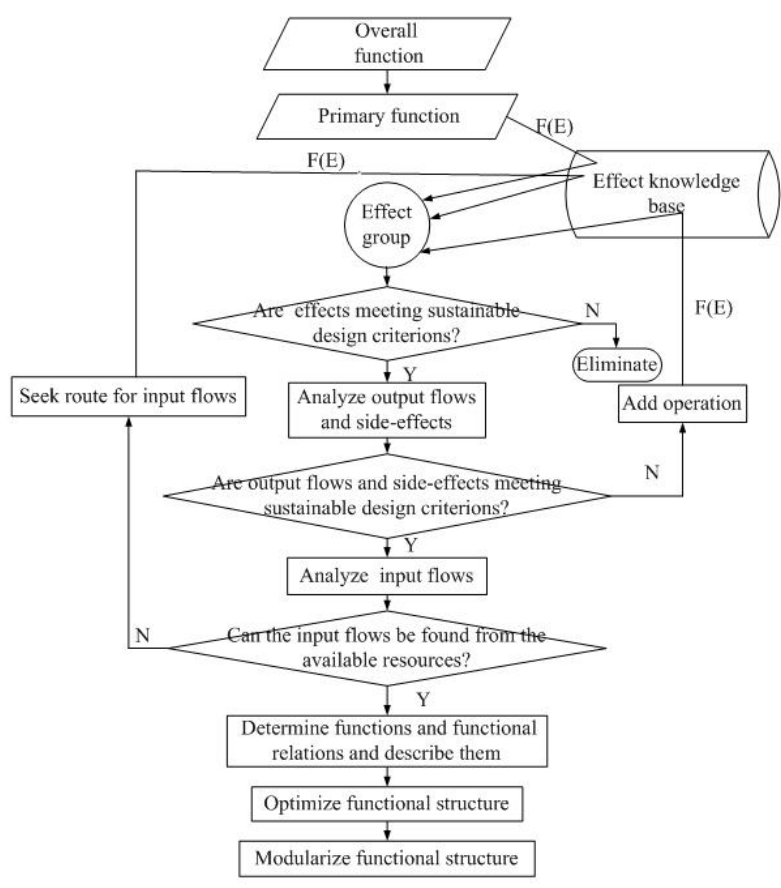

Fig. 2. Model of functional decomposition

\section{Engineering Examples}

The existing heating method is discontinuous, the efficiency of which is bad. Furthermore the heating is in settling chamber which restricts the continuity. In order to continuously deposit $\mathrm{CdTe}$, it is need to separate heating substrate from depositing $\mathrm{CdTe}$. The function of continuous heating equipment of the production line of $\mathrm{CdTe}$ thin film solar cell is continuously heating the FTO conducting glass to the required temperature in vacuum environment before the settling chamber.

The functional structure of existing model is established and is divided into several modules, as shown in Fig. 3. Fig. 4 is the functional structure of new model, which has considered the sustainability of the product and resolved the contradictions by TRIZ theory.

Functional design of continuous heating equipment confirms that the function decomposition based on existing module can search for functional structure according to technological and performance requirements, cost and simple structure only, while in the function decomposition based on the new module, designer not only according to technological and performance requirements, but also can directively select effect and analyze the sustainability of flows. So designer can consider the sustainability on the level of function and scientific principle. 


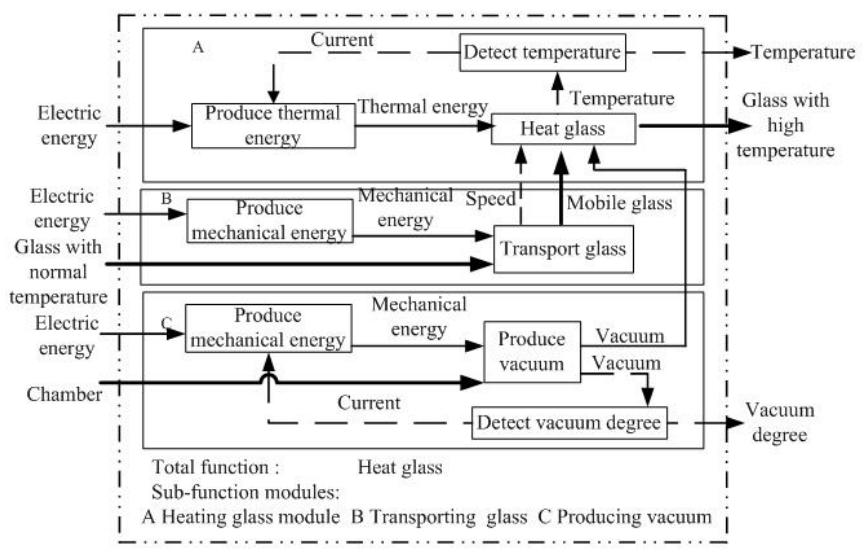

Fig. 3. Functional structure of existing model

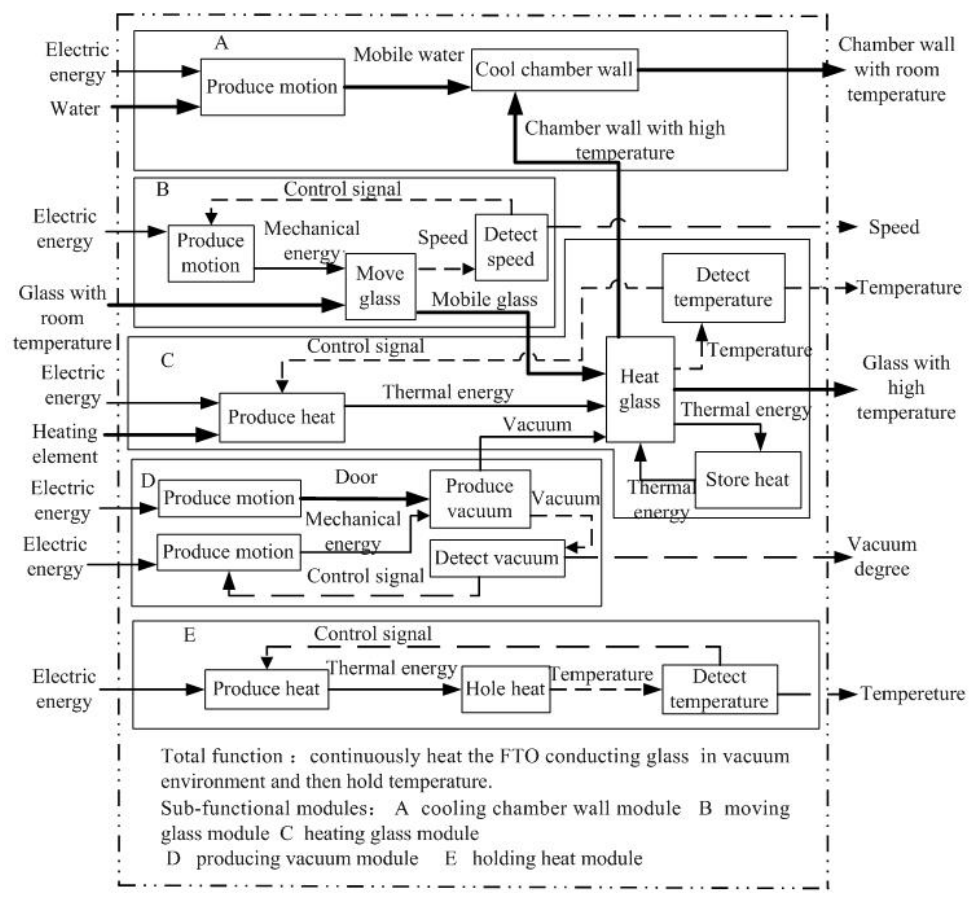

Fig. 4. Functional structure of new model 


\section{Conclusion}

Designing sustainable product is the core of producing sustainable product and the crux of sustainable development of business. In the article, puts forward sustainable design criteria for function and effect and a model of functional decomposition. In the process of function decomposition, following the sustainable design criteria can realize the purpose that considering sustainability in parallel.

Functional design of continuous heating equipment confirms that considering sustainability in process of product design in parallel, can significantly improve its sustainability.

\section{Acknowledgments}

This research is supported by the Project of Hebei Education Department (2009324, S090314), the Special Foundation for Basic Science-Technology of China (2009IM020700, 2010IM020100) and the Natural Science Foundation of China (70972050).

\section{References}

1. Value Analysis Incorporation (VAI): Value analysis, value engineering, and value management. Clifton Park 16, New York (1993)

2. Otto, K.N., Wood, K.L.: Product Design: Techniques in Reverse Engineering and New Product Development. Prentice-Hall, Upper Saddle River (2001)

3. Pahl, G., Beitz, W.: Engineering Design: A Systematic approach, 2nd edn., 3rd Printing. Springer, London (2000)

4. Liu, H., Cao, G., et al.: Product Sustainability and Realization. In: 2nd International Conference on Advances in Product Development and Reliability, pp. 790-794. Trans Tech Publications, Switzerland (2010)

5. Tan, R.: Innovative design: TRIZ Theory of Inventive Problem Solving. Mechanic industry Press, Beijing (2002)

6. Savransky, S.D.: Lesson 4 Contradictions (November 1999),

http: //www.triz-journal.com/archives/1999/11/index.htm 